\title{
Von Willebrand factor propeptide missense mutations affect anterograde transport to Golgi resulting in ER accumulation
}

\author{
Hamideh Yadegari ${ }^{1}$, Arijit Biswas ${ }^{2}$, Shariq Ahmed $^{3}$, Arshi Naz ${ }^{3}$, and Johannes Oldenburg ${ }^{1}$ \\ ${ }^{1}$ University Hospital Bonn Institute for Experimental Hematology and Transfusion \\ Medicine \\ ${ }^{2}$ Universitatsklinikum Bonn \\ ${ }^{3}$ National Institute of Blood Disease \& Bone Marrow Transplantation Karachi
}

September 24, 2020

\begin{abstract}
Von Willebrand disease (VWD), the most prevalent congenital bleeding disorder, arises from a deficiency in von Willebrand factor (VWF), which has crucial roles in hemostasis. The present study investigated functional consequences and underlying pathomolecular mechanisms of several VWF propeptide (VWFpp) variants detected in our cohort of VWD patients for the first time. Transient expression experiments in HEK293T cells demonstrated that four out of the six investigated variants (p.Gly55Glu, p.Val86Glu, p.Trp191Arg, and p.Cys608Trp) severely impaired secretion. Their co-transfections with the wt partly corrected VWF secretion, displaying loss of large/intermediate multimers. Immunostaining of the transfected HEK293 cells illustrated the retention of the VWF variants in the endoplasmic reticulum (ER). Docking of the COP I and COP II cargo recruitment proteins, ADP-ribosylation factor 1 and Sec24, onto the N-terminal VWF model (D1D2D'D3) revealed that these variants occur at VWFpp putative interfaces, which can hinder VWF loading at the ER exit quality control. Furthermore, quantitative and automated morphometric exploration of the 3-dimensional immunofluorescence images showed changes in the number/size of the VWF storage organelles, Weibel-Palade body (WPB)-like vesicles. The result of this study highlighted the significance of the VWFpp variants on anterograde ER-Golgi trafficking of VWF as well as the biogenesis of WPB-like vesicles.
\end{abstract}

\section{Introduction}

Von Willebrand factor (VWF) is a multimeric glycoprotein that plays a crucial role in primary and secondary hemostasis. Mutations in the VWF gene $(V W F)$, causing either quantitative reductions or qualitative defects of VWF, lead to von Willebrand disease (VWD), the most prevalent hemorrhagic disorder in humans . VWD is classified into three main categories. Type 1 and 3 VWD are characterized by mild/moderate reduction or virtual absence of VWF, respectively, and type $2 \mathrm{VWD}$ is characterized by functional defects in VWF . VWF is synthesized as a proVWF containing a 22 amino acid (aa) signal peptide, a 741 aa propeptide, and a 2050 aa mature VWF molecule. The proVWF is constructed as repeats in the order: D1-D2-D'-D3-A1-A2-A3-D4-C1-C2-C3-C4-C5-C6-CK . VWF monomers are dimerized in the endoplasmic reticulum (ER) by the formation of disulfide bonds between the C-terminal cysteine-knot (CK) domains of two monomers. Successively, the proVWF dimers exit the ER and are transported to the Golgi, where the $\mathrm{N}$-terminal disulfide bonds between cysteines in the D3 domains lead to the formation of multimers. The polymerization process is accompanied by proteolytic cleavage of the VWFpp (domains D1-D2) in the transGolgi network (TGN), yielding mature VWF multimers and propeptide dimers. In the endothelial cells, the VWF is either secreted directly from the TGN to the plasma membrane, which is called the constitutive secretion pathway, or it is first stored in specific organelles, Weibel-Palade bodies (WPBs), and they will be later released by the regulated exocytosis pathway . Expression of VWF in some heterologous systems, such 
as Human embryonic kidney 293 (HEK293) cells, can drive the formation of WPBs-like organelles (pseudoWPBs). The VWF multimers and the propeptide are packaged together to form secretory WPBs. The VWF propeptide (VWFpp) acts as an intracellular chaperone; it is required for both the VWF multimerization process and the trafficking from Golgi to storage vesicles . Furthermore, the propeptide region is required for the appropriate orientation of the dimeric VWF which is essential for the VWF helical assembly of tubules in nascent WPBs. Nonetheless, In the present study, we demonstrated that propeptide variants disturb the anterograde ER-Golgi trafficking via affecting VWF recruitment at the ER exit quality control.

In eukaryotic cells, trafficking of newly synthesized proteins is organized by COPI- and COPII-coated vesicles that contribute to the recruitment of proteins and ER-Golgi transport, as well as ER exit quality control (QC) by excluding misfolded cargos from vesicles . Current studies point to two major protein complexes modulating VWF exit from ER, ARF (ADP-ribosylation factors) 1-GEF and Sec23/24-Sec22b, which have a role in selecting and recruitment of the cargo proteins in COPI and COPII complexes, respectively .

In this study, we investigated the underlying pathophysiological mechanisms of six novel candidate missense substitutions located in VWFpp recognized with low VWF secretion and defects in its function in VWD patients. Four out of the six mutations (p.Gly55Glu, p.Trp191Arg, p.Asn211Asp, and p.Gly334Glu) were identified as compound heterozygous in VWD patients with non- typical phenotype (type $2 \mathrm{~A} / 2 \mathrm{~B}$ and type $1 / 2 \mathrm{~N}$ VWD) and type 3 VWD. The other two novel variations (p.Val86Glu and p.Cys608Trp) were detected as homozygous in type 3 VWD. In the present study, identified variants were reproduced and heterologously expressed to monitor intracellular trafficking, multimerization, packaging, constitutive expression, and binding functions of the VWF mutants. Furthermore, the putative mechanisms affecting exiting VWF from ER were evaluated by in silico docking of VWF with COPI and COPII recruiting proteins. The results of this study enhanced our knowledge about the biological significance of the VWFpp in health and disease.

\section{Methods}

\section{Patients and mutation analysis}

In this study, the underlying patho-molecular mechanisms VWFpp variants detected in six index patients (IPs) from Germany and Pakistan. The VWD diagnosis and classification of the IPs were made based on bleeding symptoms and standard coagulation tests, consistent with ISTH-SSC VWF guidelines. The $V W F$ analysis was before performed in Bonn Haemophilia Center, and the result of the genetic analysis, as well as coagulation tests, has been previously reported. DNA and protein sequence numbering has been done based on the reference sequence: RefSeq NM_000552.4 and NP_000543.2, respectively, and sequence variant descriptions were verified by VariantValidator online tool ( $h t t p s: / /$ variantvalidator.org/; accessed on 20.09.2020). The investigated VWF variants have been registered to the Leiden Open Variation Database (LOVD version 3.0; http://www.lovd.nl/3.0/home; submission individuals IDs: 00311186- 00311190 as well as 00311192).

\section{Constructs and cell culture}

Detected gene variants were introduced into a pMT2-VWF expression vector containing human wild-type (wt) VWF cDNA using site-directed mutagenesis . HEK 293T (DSMZ, Germany) and HEK293 (ATCC, USA) cells were cultured and transfected by lipofectamine (Life Technologies, USA), as described previously , to evaluate the constitutive secretion and intracellular localization of VWF, respectively .

\section{Quantification of VWF}

Seventy-two hours after the transfection of HEK293T cells, the supernatant medium was collected and was subsequently concentrated on Amicon Centrifugal 50K filter devices (Millipore, USA) to one-fourth of the original volume. Additionally, the transfected cells were lysed in M-per reagent buffer (Thermo Scientific, USA). The amount of VWF antigen (VWF:Ag) secreted into the medium and present in the lysate were measured using a particle-based turbidimetric assay, and the mean values $(\mathrm{n}=9)$ were expressed as a percentage of the corresponding wt. 


\section{VWF activity assays and multimer analysis}

Binding of the recombinant VWF (rWF) to platelet GPIb (VWF:GPIb) was assessed using a particle enhanced assay (Siemens Healthcare, Marburg, Germany) on a BCS XP coagulation analyzer. Furthermore, binding of the rVWF to collagen type I (VWF:CB) was determined by the ELISA method (Technoclone, Vienna, Austria) according to the manufacturer's instructions. The VWF activities were expressed as ratios to secreted VWF. rVWF multimers of concentrated secreted rVWF were analyzed by $1.6 \%$ SDS-agarose gel electrophoresis as described before.

\section{Immunofluorescence microscopy}

Transfected HEK293 cells were fixed and stained as described previously . Primary antibodies anti-VWF (Abcam, England) and anti-protein disulfide isomerase (PDI; Life technologies, USA) were used to visualize intracellular VWF and endoplasmic reticulum (ER), respectively. Z-stack images were acquired with Zeiss Apotome.2 microscopy (Carl Zeiss, Germany). The quantitative co-localization investigation was done using Zeiss ZEN 2 (blue edition; Carl Zeiss, Germany). Analysis for each set of comparisons was performed on Z-series of images of a minimum of 100 cells from two independent experiments (each in triplicate). The comparative degree of co-localization was calculated as mean Pearson's correlation coefficients.

\section{Quantification and morphometric assessment of pseudo-WPBs}

Image restoration and 3-dimensional (3D) analysis of pilled-up z-stack images (least eight fields counting a minimum of 100 cells expressing VWF for each wt and mutants) were executed by the arivis Vision4D 3.2 software (arivis AG, Imaging Science, Germany). The cells were highlighted based on edge detection algorithms followed by a smoothing preprocessing using Arivis's proprietary algorithm Blob Finder. The cells expressing VWF (green signal) were elected. Subsequently, the pseudo-WPBs were detected on the green signal after applying enhancement preprocessing tasks. The average number of pseudo-WPBs per cell was calculated by dividing the global count of the WPBs in each acquired image arena by the cells encompassing at least one WPB. The pseudo-WPBs features including, length, depth, and width (provided by measuring the longest, middle, and shortest sides of the 3D oriented bounds, respectively) as well as the shape (characterized by Sphericity factor, $\Psi$ ) and mean intensities, were computed for whole WPBs-like organelles population, including 1105 of pseudo-WPBs for wt and between 237-355 for heterozygous variants. Sphericity $(\Psi)$ describes the roundness of the $3 \mathrm{D}$ segment represented as a value between 0 and 1 , where 1 is an ideal sphere. Mean intensities show the average intensity signal value for every single pseudo-WPBs.

\section{Modeling and docking}

The N-terminal region of VWF was structurally modeled to investigate the structural-functional impact of our mutations. The D1 and D2 domains were modeled independently on the ITASSER threading server (https://zhanglab.ccmb.med.umich.edu/I-TASSER/) while assigning disulfide-bonded cysteine "Y" sulfur atom restraints, based on previously suggested disulfide combinations in these domains. The model with the best C-score from each domain was chosen as its final structure. The crystal structure of the D'-D3 assembly was downloaded from the structure database (PDB ID: 6N29; Resolution: $2.5 \AA$ ) .

The joined model of the VWFpp (D1 and D2 domains) and the D'-D3 assembly was generated using overlapping inter-domain linker regions. The linked model was subjected to a brief structure refinement simulation based on previously reported methodology . The lowest energy structure in the refinement simulation trajectory was chosen for further applications. The model's dimensions and orientation were investigated in the context of its ability to form symmetric multimers. All visualization, image rendering, and structural analysis were performed on the YASARA and Chimera platforms .

Consequently, the generated model of D1D2D 'D3 was used to dock two proteins Sec24 and ARF1, for whom limited evidence exists for their role in anterograde transport of VWF. The atomic structure of ARF1 and Sec24 was extracted from the PDB ID: 2J59 with resolution: $2.1 \AA$ and PDB ID: 3 EFO with resolution: $2.7 \AA$, respectively . All water molecules and heteroatoms including GTP, as well other molecules like peptides were removed from both structures. Both structures were refined using a previously tested simulation protocol 
before implementing docking onto the VWFpp model. In the case of Sec24, since the original structure is a complex of Sec23/Sec24, only the PDB coordinates of the recruitment protein i.e. Sec24 from the refinement simulation were used for docking. Similarly for PDB ID: 2J59, which is a complex of ARF1/ARHGAP21, only the co-ordinates of AERF1 were used for docking. The docking simulation was performedon the SwarmDock

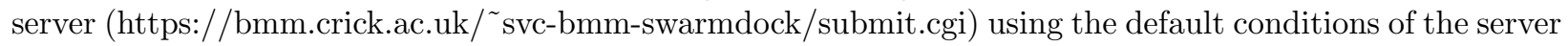
. The highest scoring docking poses $(n=10)$ from the first ten clusters based on hierarchical clustering with the democratic scoring scheme were diligently investigated especially with respect to the reported mutated residues.

\section{Statistics}

Statistical significance of the differences in data was analyzed by unpaired Student's $t$-test using GraphPad Prism version 8.0.1 (GraphPad Software, USA). The figures are presented as mean \pm SEM. A $P$ value of $<0.05$ was assumed as statistically significant.

\section{Results}

\section{Gene variants and patients}

The candidate missense variants that we have selected to study were all located in VWF propeptide (D1 and D2 domains) (Table 1). Genotype and laboratory parameters of all six patients are given in Table 1.

\section{Impact of VWFpp mutations on VWF expression and release}

To verify the impact of the detected candidate variants on basal VWF secretion, VWF:Ag levels in both supernatant and lysates of the transfected HEK293T cells were measured by a particle-based turbidimetric assay. The mean value of the constitutively secreted wt $\mathrm{rVWF}$ was $42.06 \pm 1.13 \mathrm{IU} / \mathrm{dL}$. The mutant rVWF levels are here presented as a percentage of the wt rVWF levels (Figure 1A). Four of the mutants (p.Gly55Glu, p.Val86Glu, p.Trp191Arg, and p.Cys608Trp) demonstrated severely impaired secretion. Their VWF:Ag levels in the medium were significantly lower compared to the wt, ranging from 9,8 to 10,9\% of wt (corresponding to $4.12 \pm 0.08 \mathrm{IU} / \mathrm{dL}$ to $4.58 \pm 0.20 \mathrm{IU} / \mathrm{dL}$ ) (Figure 1A, left, dark gray columns). Nonetheless, the amount of their intracellular VWF:Ag levels were not significantly different from wt (for p.Gly55Glu, p.Val86Glu, and p.Cys608Trp mutants; p >0.05) or it was even higher than that of the wt (for p.Trp191Arg), indicating intracellular retention of these mutants (Figure 1A, left, light gray columns). However, the total VWF production (sum of medium and lysate) of all these four mutants was significantly reduced. To mimic heterozygosity, co-expressions of these mutants and wt were performed. The co-expression resulted in the correction of the expression phenotype but was still $<50 \%$ of wt. The co-expressed secreted rVWF of p.Gly55Glu/wt, p.Val86Glu/wt, p.Trp191Arg/wt, and p.Cys608Trp/wt was reduced by $65 \%, 68 \%, 65 \%$, and $76 \%$, respectively, relative to the secretion of wt (Figure 1A, right).

Moreover, the variants p.Asn211Asp and Gly334Glu showed normal basal secretion compared with wt in both single and co-expression experiments ( $\mathrm{p}>0.05$ ), indicating no impact on the biosynthesis of VWF (Figure $1 \mathrm{~A})$.

\section{Description of the function and multimeric configuration of the VWF variants}

The multimer analysis revealed a range of deficiencies in the multimer structure of the secreted recombinant variants (Figure 1B). Single transfections of p.Gly55Glu, p.Val86Glu, p.Trp191Arg, and p.Cys608Trp exhibited only dimers or as well as a faint trail of tetramers (Figure 1B). The multimeric defects were only partially restored by co-transfection with wt rVWF. Multimer analysis of co-expression of wt with mutants p.Gly55Glu and p.Trp191Arg revealed a complete lack of high molecular weight multimers (HMWM), emphasizing the destructive impact of these variations on multimer assembly. Similarly, heterozygously expressed mutations p.Val86Glu/wt and Cys608Trp/wt illustrated deficiency in multimer profile but to a severer extent (loss of most of the intermediate multimers and lack of HMWM) (Figure 1B).

Functional assessment is presented as ratios of GPIb binding and VWF:CB to VWF:Ag of secreted rVWF (Figure 1C). VWF activity assays were only performed where the VWF levels in media of expressed variants 
were quantitatively adequate for reliable quantification evaluations. Functional assay results were in the concordance of the multimer structure pattern. Co-expressions of the p.Gly55Glu/wt, p.Val86Glu/wt, and p.Trp191Arg/wt showed a decrease in ratios GPIb binding and CB to VWF:Ag compared with those of wt $(\mathrm{p}<0.05)$, attributed to the loss of large/and intermediate multimers.

The secreted mutants p.Asn211Asp and Gly334Glu exhibited a complete multimer profile and normal binding activities in both single and co-expression conditions (Figure 1B and C).

\section{The causative VWFpp mutations affect the exit of VWF from ER}

Intracellular trafficking of the four rVWF mutants (p.Gly55Glu, p.Val86Glu, p.Trp191Arg, and p.Cys608Trp), causing intracellular retention of the rVWF, was investigated by immunofluorescence staining after their expression in HEK293 cells. The visual observation of the immunofluorescent images demonstrated the accumulation of the mutated rVWF within the ER (Figure 2, as well as Supplemental Videos 1-9). Further, the calculations of Pearson's coefficient confirmed an increased co-localization of mutants p.Gly55Glu, p.Val86Glu, p.Trp191Arg, and p.Cys608Trp with ER marker PDI when is compared with the wt (mean Pearson's coefficient of $0.51 \pm 0.03,0.52 \pm 0.02,0.57 \pm 0.02$, and $0.51 \pm 0.02$, respectively, vs. $0.15 \pm 0.03 ; \mathrm{P}<0.001$ ) (Figure 2A and C). Furthermore, the co-expressed VWF of p.Gly55Glu/wt, p.Val86Glu/wt, p.Trp191Arg/wt, and p.Cys608Trp/wt showed also an increased degree of co-localization compared with wt (mean Pearson's coefficient of $0.31 \pm 0.03,0.29 \pm 0.03,0.34 \pm 0.04$, and $0.32 \pm 0.03$, respectively; $\mathrm{P}<0.05$ ), but in lesser extent when they are compared with homozygously expressed variants (Figure $2 \mathrm{~B}$ and $\mathrm{C}$ ).

\section{The VWFpp mutations interfere with the quantity and morphology of the pseudo-WPBs}

A quantitative exploration of the 3D images (generated by arivis Vision4D) demonstrated a significant difference in number, size, and shape of the WPB-like vesicles within cells expressing rVWF mutants when is compared with wt (Figure $3 \mathrm{~A}-\mathrm{H}$ ). Our visual surveys ruled out the formation of any WPBs within single-transfected cells with p.Gly55Glu, p.Val86Glu, p.Trp191Arg, and p.Cys608Trp variants, consistent with their broad accumulation inside the ER as declared above. Approximately $60 \%$ of cells expressing wt VWF displayed the formation of pseudo-WPBs, with an average computed number of seven WPBs per cell, whereas only 32.6-42.9\% cells co-expressing wt plus p.Gly55Glu, p.Val86Glu, or p.Cys608Trp exhibited the formation of WPBs, with an average number of 3.5-4.0 pseudo-WPBs per cell, indicating a significant discount in generating WPBs $(\mathrm{p}<0.0001)$ (Figure 3A-C). However, the cells co-expressing wt, and p.Trp191Arg showed a significant difference in the percentage of cells forming pseudo-WPBs $(\sim 4 \%, \mathrm{p}<0.05)$, but no change in an average amount of WPBs/cell $(\sim 7.5, \mathrm{p}=0.616)$. On the other hand, quantification of the morphometric parameters of the 3D models of the pseudo-WPBs within cells coexpressing p.Trp191Arg/wt demonstrated a slight decrease in length $(2.09 \pm 0.04 \mu \mathrm{m})$ and width $(1.21 \pm 0.03 \mu \mathrm{m})$ of pseudo-WPBs compared with wt (with an average length and width of $2.18 \pm 0.09 \mu \mathrm{m}$ and $1.28 \pm 0.07 \mu \mathrm{m}$, respectively; $\mathrm{p}<0.05$ ) (Figure 3A, E, and G). Furthermore, heterozygous p.Trp191Arg/wt pseudo-WPBs displayed a slightly increased sphericity value $(0.670 \pm 0.002$ vs. $0.658 \pm 0.011$ of wt WPBs; $\mathrm{p}<0.0001)$, favoring a less elongated shape (Figure 3H). No significant changes in size (length, width, and depth) and sphericity of the pseudo-WPBs inside cells coexpressing wt and p.Gly55Glu, p.Val86Glu, and p.Cys608Trp mutants were observed when compared with wt (Figure 3A, E, F, G, and H). Nonetheless, pseudo-WPBs formed by all heterozygous mutants (with a greater extent for the p.Trp191Arg/wt) showed a substantial decline in mean intensities values of the green signals (VWF), indicating the impact of these mutants in the conformation of the storage vesicles (Figure 3D).

Structural evidence proposes that the VWFpp mutations affect the N-terminal assembly and/or VWF recruitment for anterograde transport

Our VWF N-terminal model is organized into $\mathrm{N}$ terminal globular domains D1 and D2 (VWFpp) connected to the C terminal globular D3 domain by a filamentous like $\mathrm{D}^{\prime}$ domain. The D1 and D2 domains are characterized by several regions of disorder connecting three helix-bundles to beta sheeted regions in each domain. The disordered regions are most likely instrumental in the conformational changes occurring in the course of disulfide exchange within the ER. In the present model apart from 9 cysteines in the D1- 
D2 domains, all others were observed in an oxidized form. The D3:D3 domain interaction regions in the model suggest a clear symmetry within the monomer that serves the purposes of uni-directionality for the appropriate alignment of the dimeric pro-VWF subunits and during the multimerization process (Figure 4B). Therefore the D1, D2 domain surfaces most likely serve one of the two purposes: 1) as dimeric assembly interfaces or as 2) binding region for recruitment proteins (like ARF and Sec24) during anterograde transport of VWF from ER to Golgi. The mutations on the D1, D2 domains could, therefore, affect either (N-terminal assembly/recruitment process) or both functional aspects of these domains. The docking of Sec24 and ARF1 protein on the VWF N terminal model strongly supports this idea. The docking poses/clusters of both Sec24 and ARF1 show strong similarity in spatial positioning. The majority of the high scoring docking poses for both proteins occupy the D1 domain surfaces while select few were concentrated on the D2 domain, the docking surfaces on both domains facing away from the D3:D3 domain interface (Figures 4B, 4D, and 4F). The structural alignment of the active and inactive forms of ARF-1 i.e. GTP and GDP bound structures shows a specific ARF-1 region that undergoes a conformational change (also called switch region) and therefore is the part most likely to acts as the protein recruitment surface/interface for ARF-1 (Supplemental Figure 1). In most of our observed docks, this region forms a major part of the interface between ARF 1 and VWF (Supplemental Figure 2). Interestingly, the majority of our naturally occurring mutations (3 out of 4), are located exactly on the ARF 1/ or Sec24-VWF-D1 domain interaction interface. One D2 domain mutation i.e. p.Cys608Trp does not occur directly at the interface but close to it. Since the Cys608 participates in a disulfide bond, the mutation will result in an unpaired cysteine which could significantly influence disulfide bond combinations and also disulfide exchange (especially since there exist other unpaired Cysteines in close spatial proximity). Effectively this mutation, therefore, could have potential allosteric effects on the noncovalent D1-D2 dimer interfaces or affect interaction with ARF 1 or Sec24.

\section{Discussion}

The roles of the VWFpp, D1, and D2 domains, in both multimer assembly and storage of VWF in WPBs, are well established. Each of the two D1 and D2 domains contains a CGLC amino acid motif which is considered to be a consensus sequence of the PDI enzyme family, having a catalytic role in N-terminus interchain disulfide bonds between the D3 domains to form large multimers in the Golgi compartments . Furthermore, the propeptide serves as an intramolecular chaperone, aligning the dimeric pro-VWF subunits in the appropriate special alignment to facilitate interdimer N-terminal disulfide bond formation during multimerization and to ensure VWF helical assembly into tubules in nascent WPBs. Another probable function attributed to the VWFpp is the sorting of the mature VWF into storage granules via an intrinsic signal (linear sequence or conformation) in the trans-Golgi .

Nonetheless, here in this study, we demonstrated that VWFpp missense variants hamper the exit of VWF from the ER, resulting in VWF sequestration in the ER compartment. The present study showed that four out of the six VWFpp variants (p.Gly55Glu, p.Val86Glu, p.Trp191Arg, and p.Cys608Trp) severely impaired secretion, along with intracellular retention of these variants. Further, we illustrated that VWF proteins with the mutations residing in propeptide are accumulated in the ER, and do not appear to generate WPBs-like granules. We demonstrated an increased co-localization of these VWF mutants with the luminal ER marker, PDI, when is compared with wt.

Multiple QC mechanisms operate within the ER to ensure fidelity of protein expression in cells. These mechanisms, in general, rely on a repertoire of molecular chaperones and protein folding facilitators that promote correct protein folding and detect defective proteins for degradation. Additionally, ER-Golgi export QC is another checkpoint that ensures the secretion of correctly folded proteins through cargo receptors. By recruiting cargos for specific capture into COP I and COP II vesicles, cargo receptors can monitor the folding status of their clients before capturing into vesicles . Recently, Lopes-da-Silva et al showed that the ARF family of proteins contributes to the ER-Golgi trafficking of the VWF . In the active state, when bound to GTP, ARFs initiate coat and cargo recruitment onto intracellular membranes to conduct anterograde transportation . The switch between inactive and active state occurs through GTP/GDP exchange/binding that is mediated by guanine nucleotide exchange factors (GEFs). Lopes-da-Silva et al demonstrated that GBF1 
(one of the guanine nucleotide exchange factors) reduction affects the rate of VWF movement between ER and Golgi, indirectly implicating the ARF family of proteins as the major recruitment proteins for anterograde transport of VWF . Another recent study demonstrated that the rate of the anterograde transport between ER and Golgi and consequently the morphology of the WPBs is dependent on the Sec22b-containing SNARE complexes. Sec22b is a component of ER-derived COPII-coated vesicles that direct proteins from ER to the Golgi, and interacts with Sec23/Sec24 of the COPII coating complex, which is essential for cargo recruitment in the ER. Both Sec22b silencing and Sec24 deficiency were reported to result in ER retention of the secretory proteins. Since our microscopic analysis suggested the accumulation of VWF mutants in ER, we tested the hypothesis that the misfolded VWF hampers its recruitment into COP I and COP II vesicles (mediated by ARF or Sec24) by docking one of the active ARF protein (GTP bound ARF1) and Sec24 structures onto our N-terminal VWF model. The fact that most of the variants occur at the ARF1/or Sec24-VWFpp putative interfaces on these docks strongly support the idea that the ER accumulation might be a result of these mutations interfering with the ARF/or Sec24-VWF recruitment process for anterograde transport. Nevertheless, we propose this mechanism as a putative affected QC pathway, and we can not deny involvement of the other QC checkpoints. Therefore, our structural analysis results will need to be verified by more experimental evidence in the future.

Accumulation of VWF mutants in ER was associated with reduced intracellular packaging and defect in secretion of VWF. Nonetheless, the homozygous expression of these propeptide variants still showed a minor quantity of secreted VWF, only $10 \%$ of wt, as well as a trail of the oligomers (i.e. tetramers). This suggests that a small number of dimers still succeed to rescue from the ER and reach their destination in trans-Golgi. Further, all of the four causative variants showed a $65 \%-75 \%$ fall in the secretion of VWF when heterozygously expressed, indicating their dominant impact on secretion, along with an increased colocalization with the ER marker (though with a lesser extent compared with homozygously expressed variants). Besides, the co-expression of these VWFpp variants with wt revealed a deficiency in VWF multimer assembly, lacking large/and intermediate multimers. We consequently assume that the limited number of the mutant homodimers/or heterodimers rescued from the ER will successfully cross-over to the Golgi and will interfere with the accurate orientation of the propeptide within the dimeric bouquet and succeeding multimer elongation, based on our observations made on the VWF N-terminal model's orientation.

Furthermore, in the current study, the biogenesis of WPBs in co-expressing cells of wt/mutants was explored by computerized morphometric analysis of the whole pseudo-WPBs population using state-of-the-art arivis Vision4D. Initially, microscopic inspections revealed a reduction in the fraction of the cells generating pseudoWPBs in cells co-expressing wt/mutants. The computed average number of pseudo-WPBs per cell was $\sim 7$ for cells expressing wt, this number was less for the cells co-expressing p.Gly55Glu, p.Val86Glu, and p.Cys608Trp ( 3.4-4.0 per cell). Altogether, both declines in the portion of cells forming pseudo-WPBs and their average number per cell for the later co-expressed mutants indicate their dominant-negative impact on the formation of the storage vesicles. The reduction in the number of WPBs was previously also reported for the mutations detected in type 1 and type $2 \mathrm{~A}$ VWD, which was associated with intracellular VWF retention and decreased secretion. However, these previous reports were based on only visual inspections but not the automated quantifications as we have done in the current study.

The WPBs have a distinct rod-shaped morphology and their length varies between 0.5 and $5 \mu \mathrm{m}$ in endothelial cells. Karampini et al have quantified the length of WPBs in cultured endothelial cells (HUVECs) and they reported the average length of $\sim 2.2 \mu \mathrm{m}$. Furthermore, in our lab, quantification WPBs length in the blood outgrowth endothelial cells (BOECs) showed the various WPBs lengths with an average length of $2.281 \mu \mathrm{m}$ \pm 0.01 (data not shown). Likewise, here, the quantitative analysis of the whole pseudo-WPBs population in HEK293 cells showed various sizes of pseudo-WPBs (Figure 3E) with an average length of $2.180 \pm 0.09 \mu \mathrm{m}$ in cells expressing wt. The co-expressed p.Trp191Arg caused a reduction in both length and width of the WPBslike granules. It showed also slightly augmented sphericity, which indicates the storage granules have a more rounded shape than elongated. Although co-expression of the p.Gly55Glu, p.Val86Glu, and p.Cys608Trp seems not to affect the size of pseudo-WPBs, the reduction in the computed mean intensity values implies unspecified changes in their configuration, besides their influence on the number of the generated pseudo- 
WPBs. Variants p.Asn211Asp and p.Gly334Glu did not demonstrate any defect in rVWF expression and function in vitro. Based on our observations, we suggest these two variants should be considered as benign variants rather than pathologic mutations.

In sum, here in this study, we demonstrated that VWFpp mutations hinder anterograde ER-Golgi VWF export, resulting in ER accumulation. Consequently, retention of the VWF inside the ER resulted in no storage vesicle formation in the homozygously transfected cells, and a decrease in the number of generated pseudo-WPBs in heterozygously transfected cells. Furthermore, the heterozygous VWFpp mutants cause changes in the size and configuration of the WPBs-like granules, with variability in the extent/type of these changes. This highlights the consequence of the VWF variants on biogenesis and configuration of WPBs, which in turn may affect the recruitment of the other WPBs' cargos.

\section{Authorship and conflict-of-interest statements}

Contribution: H.Y. designed the study; performed the experimental work; interpreted the data; wrote the paper. A.B. designed and performed molecular modeling and structural analysis. S.A. and A.N. did DNA sequence analyses and contributed to the patient's data. J.O. supervised the study, reviewed and edited the manuscript. The authors have no conflicts of interest.

\section{Data availability statement}

The data that support the findings of this study are available from the corresponding author upon reasonable request.

\begin{tabular}{|c|c|c|c|c|c|c|c|c|}
\hline Patients & $\begin{array}{l}\text { VWD } \\
\text { Type }\end{array}$ & $\begin{array}{l}\text { Nucleotide } \\
\text { Ex- } \\
\text { change } \\
\end{array}$ & $\begin{array}{l}\text { AA } \\
\text { Ex- } \\
\text { change }\end{array}$ & Domain & $\begin{array}{l}\text { VWF:Ag } \\
\text { IU/dL }\end{array}$ & $\begin{array}{l}\text { VWF:Rco } \\
\text { IU/dL }\end{array}$ & $\begin{array}{l}\text { FVIII:C } \\
\text { IU/dL }\end{array}$ & $\begin{array}{l}\text { Multi } \\
\text { Pat- } \\
\text { tern }\end{array}$ \\
\hline IP-1 & $2 \mathrm{~A} / 2 \mathrm{~B}$ & $\begin{array}{l}\text { c. } 164 G>A \\
\text { c. } 4019 \mathrm{~T}>\mathrm{G}\end{array}$ & $\begin{array}{l}\text { p.Gly55Glu } \\
\text { p.Leu1340Arg }\end{array}$ & $\begin{array}{l}\text { D1 } \\
\text { (VWD1) } \\
\text { A1 }\end{array}$ & 12 & 7 & 42 & $\begin{array}{l}\text { Loss of } \\
\text { large } \\
\text { multim } \\
\text { normal } \\
\text { triplet } \\
\text { structu }\end{array}$ \\
\hline IP-2 & 3 & $c .257 T>A$ & p. Val86Glu & $\begin{array}{l}\text { D1 } \\
\text { (VWD1) }\end{array}$ & 1 & 1 & 3 & $\begin{array}{l}\text { No } \\
\text { multim }\end{array}$ \\
\hline IP-3 & $2 \mathrm{~A} / 2 \mathrm{~B}$ & $\begin{array}{l}c .571 T>C \\
\text { c. } 3797 \mathrm{C}>\mathrm{T}\end{array}$ & $\begin{array}{l}\text { p.Trp191Arg } \\
\text { p.Pro1266Leu }\end{array}$ & $\begin{array}{l}\text { D1 } \\
\text { (VWD1) } \\
\text { A1 }\end{array}$ & 29 & $16^{*}$ & 74 & $\begin{array}{l}\text { Loss of } \\
\text { large } \\
\text { multim } \\
\text { increas } \\
\text { abunda } \\
\text { of the } \\
\text { fastest } \\
\text { migrat } \\
\text { band ir } \\
\text { triplet } \\
\text { structu }\end{array}$ \\
\hline IP-4 & 3 & $\begin{array}{l}\text { c. } 631 A>G \\
\text { c. } 3931 \mathrm{C}>\mathrm{T}\end{array}$ & $\begin{array}{l}\text { p.Asn211Asp } \\
\text { p.Gln1311X }\end{array}$ & $\begin{array}{l}\text { D1 (C8-1) } \\
\text { A1 }\end{array}$ & 1 & 2 & 3 & $\begin{array}{l}\text { No } \\
\text { multim }\end{array}$ \\
\hline IP-5 & $1 / 2 \mathrm{~N}$ & $\begin{array}{l}\text { c. } 1001 G>A \\
\text { c. } 970 \mathrm{C}>\mathrm{T} \\
\text { c. } 2561 \mathrm{G}>\mathrm{A}\end{array}$ & $\begin{array}{l}\text { p.Gly334Glu } \\
\text { p.Arg324X } \\
\text { p.Arg854Gln }\end{array}$ & $\begin{array}{l}\text { D1 (TIL1) } \\
\text { D1 (TIL1) } \\
\text { D' }^{\prime}\left(\mathrm{E}^{\prime}\right)\end{array}$ & 35 & 39 & 22 & Norm \\
\hline IP-6 & 3 & c. $1824 C>G$ & p.Cys608Trp & $\begin{array}{l}\mathrm{D} 2 \\
(\mathrm{C} 8-2)\end{array}$ & 1 & 1 & 4 & $\begin{array}{l}\text { No } \\
\text { multim }\end{array}$ \\
\hline
\end{tabular}


Table 1. Summary of mutation and phenotype characteristics of the patients.

IP, Index patient; VWD, von Willebrand disease; VWF, von Willebrand factor; VWF:Ag, VWF antigen; VWF:RCo, Ristocetin cofactor activity; FVIII:C, factor VIII coagulant activity. Variants with underline indicate the VWF propeptide variants studied here.* For IP-3, instead of VWF:RCo, VWF binding to platelet GPIb (VWF:GPIb) is reported.

The VWF DNA and protein reference sequences are RefSeq NM_000552.4 and NP_000543.2, respectively.

\section{Figure Legends}

Figure 1. Impact of the VWF propeptide mutations on secretion, function and multimer structures of the VWF protein. (A) HEK293T cells were transiently transfected with rVWF-wt, p.Gly55Glu, p.Val86Glu, p.Trp191Arg, p.Asn211Asp, p.Gly334Glu and p.Cys608Trp (left side); the mutant constructs were co-transfected with VWF-wt at a 1:1 ratio (right side). Recombinant VWF antigen levels (VWF:Ag) in medium (dark gray bars) and lysate (light gray bars) were measured and expressed as a percentage relative to the VWF-wt. Each bar represents the average value and \pm SEM of three independent experiments in triplicate.(B) The VWF binding activities, VWF:GPIb and VWF:CB. The functional assessments are presented as mean and \pm SEM of ratios VWF:GPIb and VWF:CB to VWF:Ag from three independent experiments in triplicate. The $\mathrm{n} / \mathrm{a}$ indicates where the amount of mutated VWF antigen in media was not adequate for reliable quantification evaluations.(C) The multimer pattern of rVWF-wt and mutants in the medium of transfected HEK293T cells (both single and co-transfected cells) analyzed by electrophoresis on 1.6\% SDS-agarose gel. Lines 1 and 2 show normal pool plasma and rVWF-wt, respectively. Lines 3, 5, 7, 9, 11 and 13 represent single-expressed VWF mutants, whereas lines 4, 6, 8, 10, 12 and 14 illustrate co-expressed mutants with VWF-wt in a ratio of 1:1.

wt, wild type; VWF, von Willebrand factor;rVWF, recombinant VWF; VWF:Ag, VWF antigen;VWF:GPIb, VWF binding to platelet GPIb; VWF:CB, VWF binding to collagen type I.

Figure 2. Intracellular localization and storage of recombinant VWF (rVWF) variants in transfected HEK293 cells. (A and B) rVWF-wt and pathogenic VWF variants were expressed in HEK293, subsequently, intracellular trafficking and storage of VWF were investigated. Cells were fixed and immunostained to visualize VWF (green, left channels) and endoplasmic reticulum (ER) using the PDI marker (red, middle channels). Right channels illustrate the merge of green and red staining, showing colocalization of the two proteins VWF and PDI. The punctate staining (green) represents a storage of VWF in pseudo-WPBs, whereas the diffuse green staining indicates VWF retained in ER. Scale bar $=10 \mu \mathrm{m}$. (A) HEK293 cells were transiently transfected with rVWF-wt or the pathogenic VWF variants p.Gly55Glu, p.Val86Glu, p.Trp191Arg, and p.Cys608Trp. (B) HEK293 cells were co-transfected with VWF mutant and wt in a 1:1 ratio to imitate heterozygous expression. (C) Degree of co-localization of VWF mutants with ER. This bar graph represents the mean of Pearson's coefficient for rVWF-wt and pathologic variants (single and co-expressed) analyzed in this study. Error bars represent the SEM. The Pearson's coefficient has been calculated from at least 100 cells for each construct on the Zeiss ZEN 2 (blue edition) software.

Figure 3. Immunostaining and quantitative morphological analysis of WPBs-like vesicles in cotransfected HEK293 cells. (A)Immunofluorescence images show a 3D model of formation of the WPBslike granules in transiently transfected HEK293 cells with recombinant VWF wt as well as transiently cotransfected wt with variants p.Gly55Glu, p.Val86Glu, p.Trp191Arg, and p.Cys608Trp in a 1:1 ratio imitating heterozygous expression. Scale bar $=10 \mu \mathrm{m}$. Boxes represent close-up views of the processed 3D models of the WPBs-like granules surrounding with oriented bounds which are generated by arivis Vision4D 3.2 software. The size measurements (length, depth, and width) of some wt and mutant pseudo-WPBs are provided jus as examples; the sizes are presented as $\mu \mathrm{m}$ (B) The fraction of cells forming WPBs-like granules are presented as \% of the cells detected with green signal (indicating expressing VWF) after inspecting at least 100 cells for each HEK293 cells transfected with wt VWF and HEK293 cells co-transfected with wt and VWF variants. Bars represent means \pm SEM from a minimum of eight fields of view. (C) The relative average number of WPBs-like organelles per cell in transfected HEK293 cells, calculated by dividing the 
global count of the WPBs in each acquired image arena by the cells encompassing at least one WPB (D) The mean intensity signal value (green, VWF) for every single WPB-like organelle was calculated. Bars display average \pm SEM from the whole population of WPBs-like granules (hundreds of WPBs). (E, F, and G) panels demonstrate the average value of the longest (length), middle (depth), and shortest sides (width) from the whole population of WPBs-like granules (hundreds of WPBs), respectively. Each bar represents the average value and \pm SEM. $(\mathbf{H})$ The Sphericity factor $(\Psi)$, describing the roundness of the 3D granules were computed. It is represented as a value between 0 and 1 , where 1 is an ideal sphere. Each value implies the average rate \pm SEM of the whole population of WPBs-like granules.

ns: non-significant; *: $\mathbf{P}<\mathbf{0 . 0 5}$; ** $\mathbf{P}<\mathbf{0 . 0 0 2}$; ***: $\mathbf{P}<\mathbf{0 . 0 0 0 1}$ by Student t-test.

Figure 4. The generated merged model of the D1-D2-D'-D3 domains and its docking with ARF1 protein and Sec24. (A) The structural model represents the joined models of the D1-D2-D'-D3 domains. D1 and D2 domains (VWFpp) models (orange and green, respectively) were generated individually on the ITASSER threading server, and the crystal structure of the $\mathrm{D}^{\prime}$-D3 assembly (cyan and yellow, respectively) was downloaded from the structure database (PDB ID: 6N29; Resolution: $2.5 \AA$ ). Subsequently, the joined model of the VWFpp and the D'-D3 assembly was created. All propeptide cysteines are represented in blue colored stick format. The mutated residues have been depicted with their molecular surface, and are colored red. (B) The 3-dimensional model illustrates an orientational depiction of the joined model of the propeptide and the D'-D3 domains. The model itself is depicted by its molecular surface area. The molecular surface area of the linearly contiguous D1,D2,D' and D3 domains are colored in a darker shade of grey while the D3 domain of an adjacent dimer and part of the complete model is colored in a lighter shade of grey. (C) The schematic illustration of the multimeric arrangement and tubular packing of the N-terminal of the VWF. In the ER, VWF monomers dimerized through disulfide bonds formation between C-terminal cysteineknot (CK) domains, later in trans-Golgi each pro-VWF dimer zip up into a dimeric bouquet (transparent light blue boxes display the arrangement of the D1 (orange) D2 (green) D' (cyan) D3 (yellow) domains in each dimeric bouquet). Forming the dimeric bouquets are crucial for accurate VWF dimer incorporation into growing tubules inside WPBs. Homotypic interactions between the D1 and D2 domains (non-covalent dimerization of N-terminal ends within proVWF dimeric bouquet, shown with broken arrow) places one D 'D3 domain within a dimer apart from one another, and brings one D'D3 nearby to one D'D3 domain from another assembled dimer, facilitating N-terminal disulfide bond between dimers . (D) panel shows the ten best docking poses resulting from docking the crystal structure of ARF1 on our propeptide model. The backbone of both participating proteins is depicted in ribbon format with ARF1 colored magenta and VWF colored grey. The residues on which causative mutations have been reported in our study have been depicted with their molecular surface are colored red. (E) panel shows a close-up view of the four mutated residues reported in our study on the model, especially for their position vis-à-vis the ARF1 interaction interfaces (residues Gly55, Val86, Trp191) and also free unpaired Cysteines (residue Cys608). All protein structures are depicted in ribbon format, with VWF colored grey and ARF-1 colored magenta. The regions on ARF-1 which undergoes a conformational change during its transition from an inactive to an active state (upon GTP binding) are colored yellow. The mutated wild type residues are depicted in their stick formats. The wild type residues of Gly55, Val86, and Trp191 are colored red. The Cysteines forming disulfide bonds are also depicted in blue colored stick format, while the unpaired cysteines are depicted in green colored stick formats. (F) panel shows the ten best docking poses resulting from docking the crystal structure of Sec24 on our propeptide model. The backbone of both participating proteins is depicted in ribbon format with Sec24 colored magenta and VWF colored grey. The residues on which causative mutations have been reported in our study have been depicted with their molecular surface are colored red. (G) panel shows a close-up view of the four mutated residues reported in our study on the model, especially for their position vis-à-vis the Sec24 interaction interfaces (residues Gly55, Val86, Trp191 and Cys608). 


\section{Figure 1}

A

C

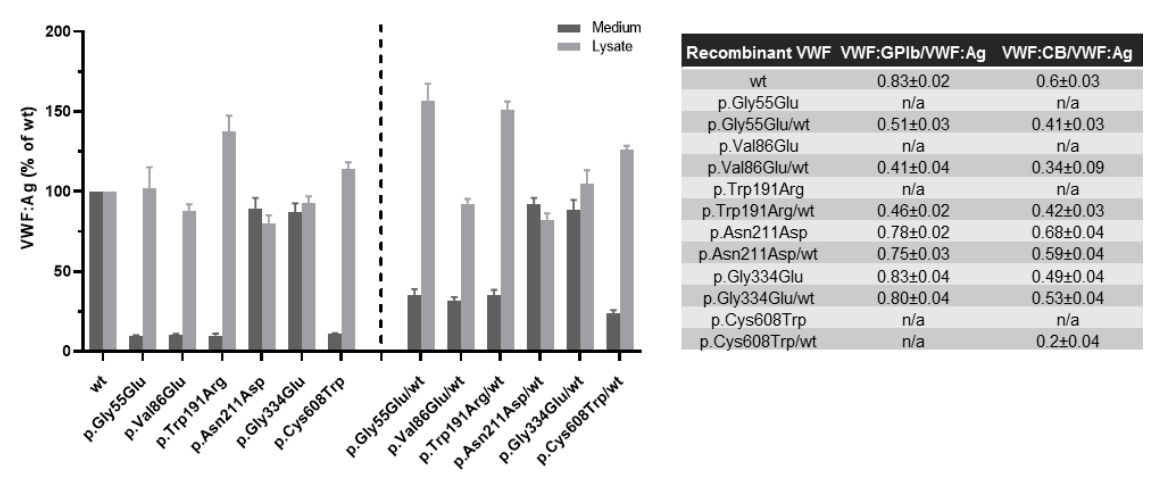

B

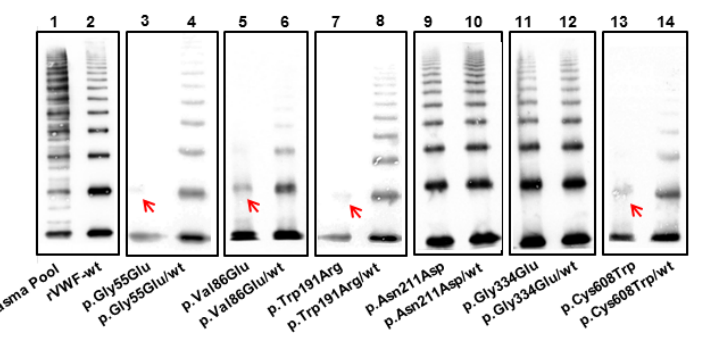


Figure 2

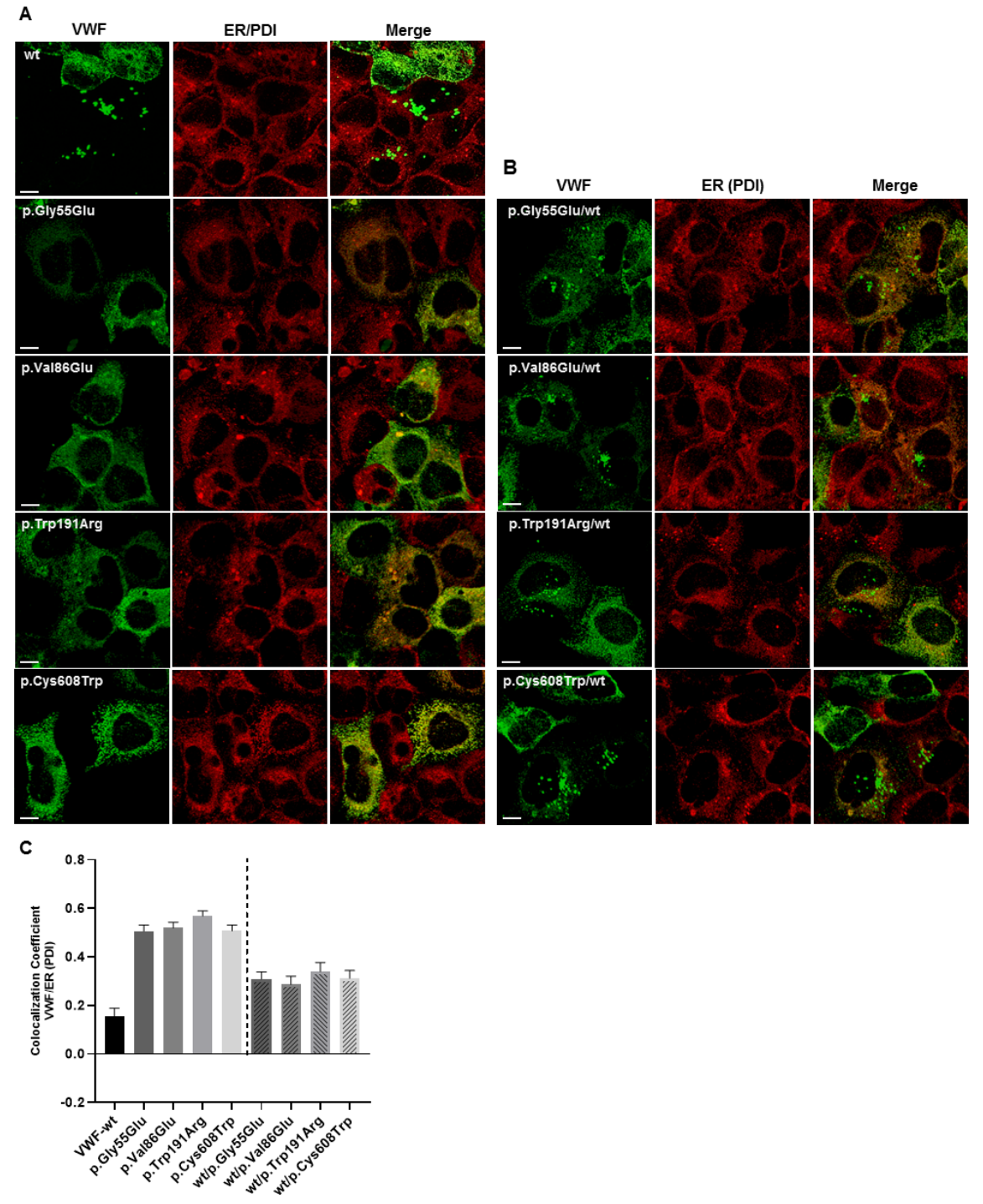


Figure 3

A
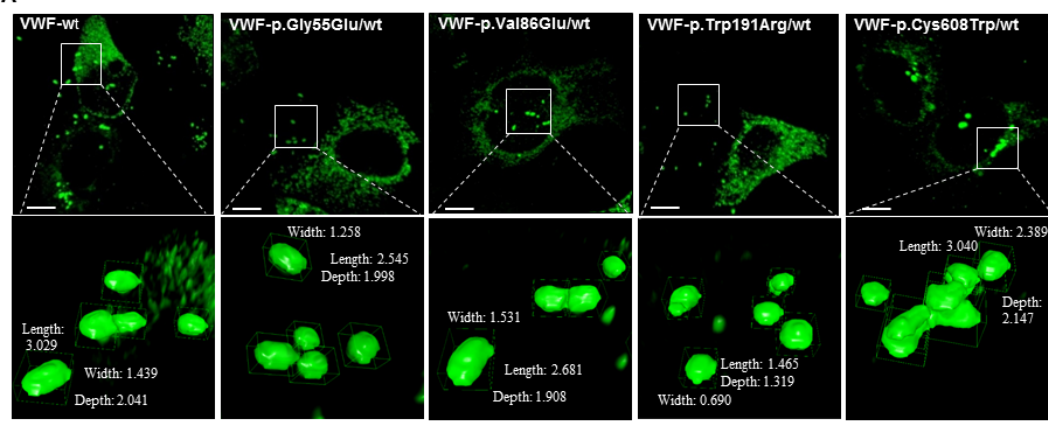

B

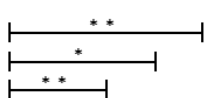

c

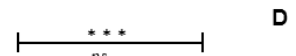

D
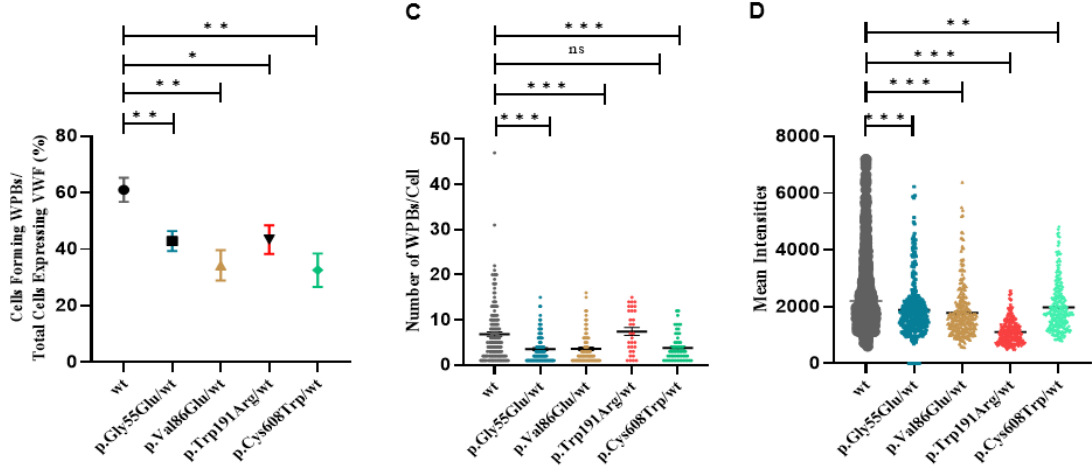

E
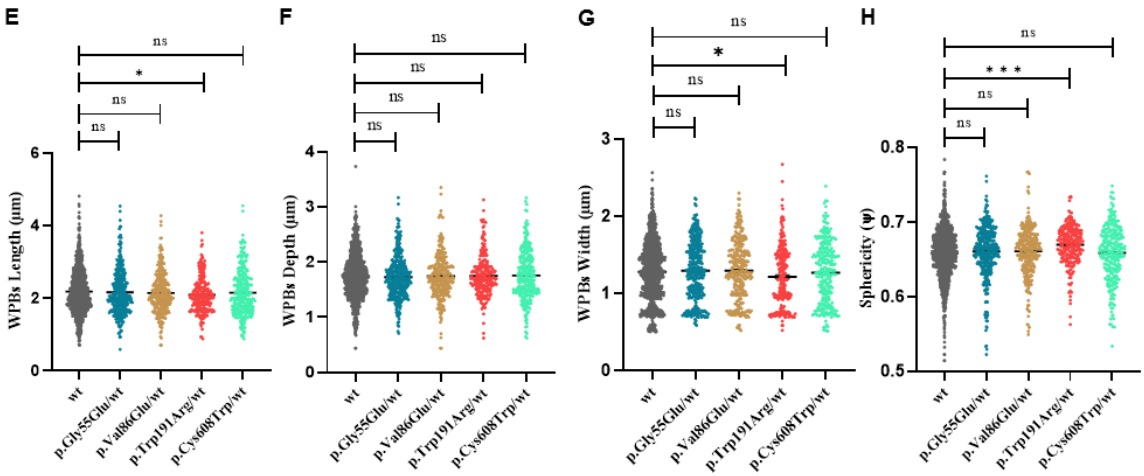


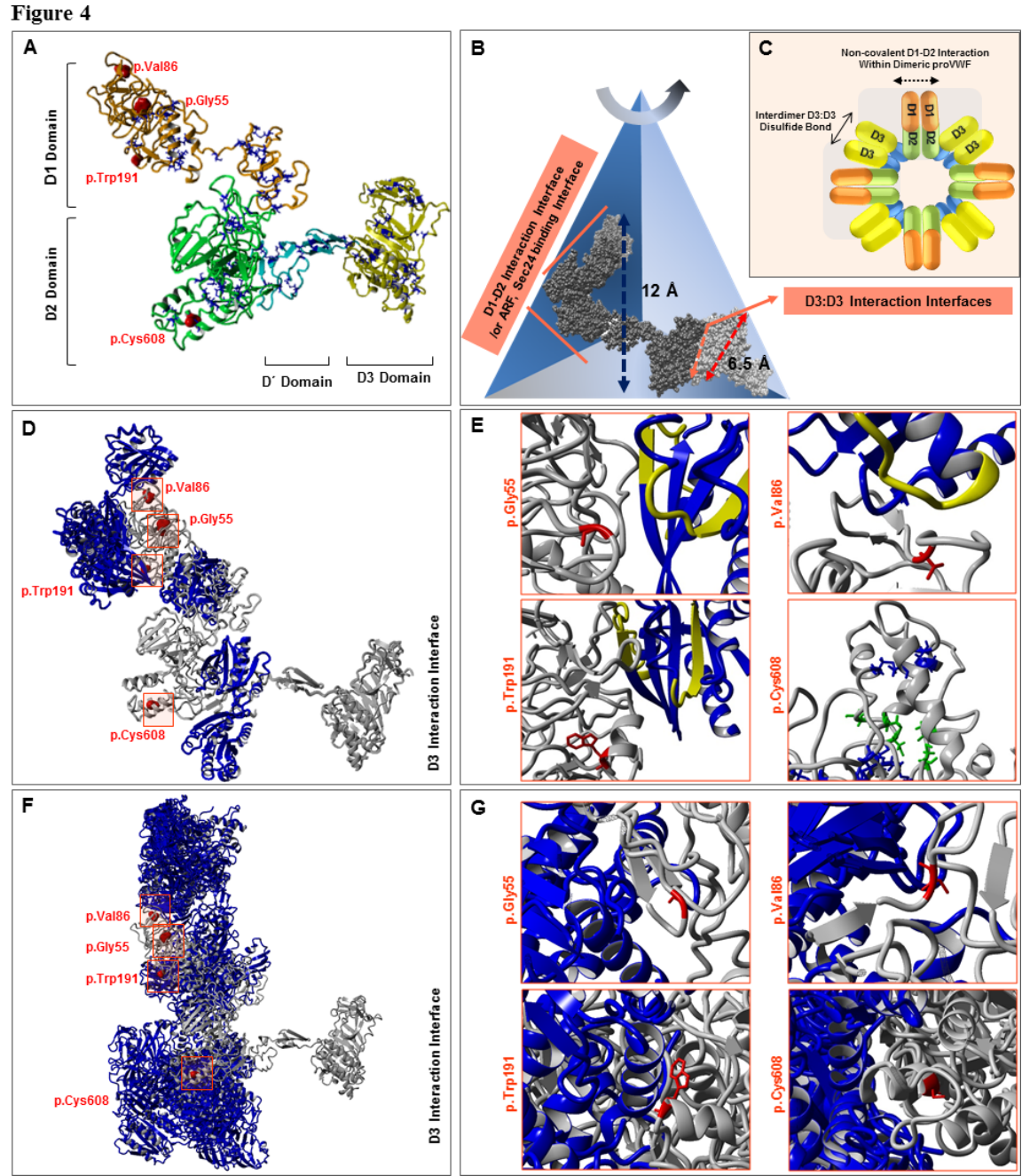

